Celine C. Corona, Man Zhang, Abhishek Wadhawan, Melanie L. Daue, Maureen W. Groer, Aline Dagdag, Christopher A. Lowry, Andrew J. Hoisington, Kathleen A. Ryan, John W. Stiller, Dietmar Fuchs, Braxton D. Mitchell, Teodor T. Postolache*

\title{
Toxoplasma gondii IgG associations with sleep- wake problems, sleep duration and timing
}

https://doi.org/10.1515/pteridines-2019-0001

received December 31, 2018; accepted January 29, 2019.

Abstract: Background: Evidence links Toxoplasma gondii (T. gondii), a neurotropic parasite, with schizophrenia, mood disorders and suicidal behavior, all of which are associated and exacerbated by disrupted sleep. Moreover, low-grade immune activation and dopaminergic overstimulation, which are consequences of $T$. gondii infection, could alter sleep patterns and duration.

Methods: Sleep data on 833 Amish participants [mean age $(\mathrm{SD})=44.28$ (16.99) years; 59.06\% women] were obtained via self-reported questionnaires that assessed sleep problems, duration and timing. T. gondii IgG was measured with ELISA. Data were analyzed using multivariable logistic regressions and linear mixed models, with adjustment for age, sex and family structure.

Results: T. gondii seropositives reported less sleep problems $(p<0.005)$ and less daytime problems due

*Corresponding author: Teodor T. Postolache, Mood and Anxiety Program, Department of Psychiatry, University of Maryland School of Medicine, Baltimore, MD 21201, USA; Rocky Mountain Mental Illness Research Education and Clinical Center (MIRECC), Veterans Integrated Service Network (VISN) 19, Military and Veteran. Microbiome: Consortium for Research and Education (MVM-CoRE), Aurora, CO 80045, USA; Mental Illness Research, Education and Clinical Center (MIRECC), Veterans Integrated Service Network (VISN) 5, VA Capitol Health Center Network, Baltimore, MD 21201, USA, E-mail: tpostola@som.umaryland.edu

Celine C. Corona, Abhishek Wadhawan, Aline Dagdag, John W. Stiller: Mood and Anxiety Program, Department of Psychiatry, University of Maryland School of Medicine, Baltimore, MD 21201, USA

Man Zhang, Melanie L. Daue, Kathleen A. Ryan, Braxton D. Mitchell: Division of Endocrinology, Diabetes and Nutrition, Department of Medicine, University of Maryland School of Medicine, Baltimore, MD 21201, USA

Abhishek Wadhawan: Saint Elizabeths Hospital, Psychiatry Residency Program, Washington, DC 20032, USA

Melanie L. Daue, Kathleen A. Ryan, Braxton D. Mitchell: Program for Personalized and Genomic Medicine, University of Maryland School of Medicine, Baltimore, MD 21201, USA

Melanie L. Daue, Braxton D. Mitchell: Geriatrics Research and Education Clinical Center, Veteran Affairs Medical Center, Baltimore, MD 21201, USA to poor sleep $(p<0.005)$. Higher $T$. gondii titers were associated with longer sleep duration $(p<0.05)$, earlier bedtime $(p<0.005)$ and earlier mid-sleep time $(p<0.05)$.

Conclusions: It seems unlikely that sleep mediates the previously reported associations between $T$. gondii and mental illness. Future longitudinal studies with objective measures are necessary to replicate our findings.

Keywords: IgG, mid-sleep time, sleep duration, sleep timing, Toxoplasma gondii.

\section{List of abbreviations}

ELISA: Enzyme-linked immunosorbent assay

GABA: Gamma-aminobutyric acid

IgG: Immunoglobulin G

IL: Interleukin

Maureen W. Groer: College of Nursing, University of South Florida College of Nursing, Tampa, FL 33612, USA

Christopher A. Lowry: Department of Integrative Physiology and Center for Neuroscience, University of Colorado Boulder, Boulder, CO 80309, USA; Department of Physical Medicine and Rehabilitation and Center for Neuroscience, University of Colorado Anschutz Medical Campus, Aurora, CO 80045, USA

Christopher A. Lowry, Andrew J. Hoisington: Rocky Mountain Mental Illness Research Education and Clinical Center (MIRECC), Veterans Integrated Service Network (VISN) 19, Military and Veteran. Microbiome: Consortium for Research and Education (MVM-CoRE), Aurora, CO 80045, USA

Andrew J. Hoisington: Department of Systems Engineering and Management, Air Force Institute of Technology, Wright-Patterson AFB, OH 45433, USA

John W. Stiller: Saint Elizabeths Hospital, Department of Neurology, Washington DC 20032, USA; Maryland State Athletic Commission, Baltimore, MD 21202, USA

Dietmar Fuchs: Division of Biological Chemistry, Biocenter, Innsbruck Medical University, Innsbruck, Austria

Aline Dagdag: Psychiatry Adult Inpatient \& Behavioral Health, University of Maryland Medical Center, Baltimore, MD 21201, USA 
NREM: Non-rapid eye movement

T. gondii: Toxoplasma gondii

TNF: Tumor necrosis factor

\section{Introduction}

During infection, sleep and the immune system interact to improve the fitness of the host by better allocation of energy to critical physiological functions implicated in controlling the infection [1]. While the majority of studies focus on viral and bacterial infections, sleep is strongly altered in parasitic infections too. For instance, in the human African trypanosomiasis (sleeping sickness), there is a complete loss of circadian rhythm with narcolepticlike fits and decreased total hours of wakefulness [2]. Moreover, changes in sleep duration have been associated with parasitic infection in 12 mammalian species [3]. It has been suggested that sleep "fuels" the immune system to increase resistance to parasites [4]. Levels of cytokines such as interleukin (IL)-1 1 , IL-10, IL-12 and tumor necrosis factor (TNF) are at their highest during sleep, regardless of circadian rhythms [1]. Proinflammatory cytokine activity increases when a host is faced with an immune challenge, which leads to increased duration of non-rapid eye movement (NREM) sleep [4]. White blood cell counts have also been associated with the total sleep time in 26 mammalian species [3].

Toxoplasma gondii ( $T$. gondii) is a protozoan parasite estimated to have infected 30\% of the world's population [5]. Among Americans, the prevalence of $T$. gondii seropositivity is approximately $13.2 \%$ [6]. It has the ability to invade numerous warm-blooded animals as intermediate hosts and members of the feline family, who are its definitive hosts [7]. Within the cat, T. gondii reproduces sexually and oocysts are formed and excreted into the environment where they are ingested by rodents and other intermediate hosts, including humans, through contamination of food and water [8]. Consuming contaminated undercooked meat also infects humans [9-13]. The environment, the host's genetic framework, the specific strain of $T$. gondii, the parasitic stage and the route of contamination during inoculation, all contribute to the severity of $T$. gondii infection [14]. Many parasites [15], including T. gondii $[16,17]$, have the capability to change the behavior of their hosts to enhance the completion of their life cycle. The immune system of an immunocompetent host with chronic $T$. gondii infection contains the parasite to its slow-growing forms inside tissue cysts [18-20]. There has been growing evidence that suggests increased rates of $T$. gondii infection in individuals with mental illness, in particular schizophrenia, including new-onset schizophrenia [21, 22]. Sutterland et al. (2015) performed a meta-analysis and uncovered significant associations of $T$. gondii infection with schizophrenia, bipolar disorder and obsessive-compulsive disorder [23]. Recently, we reported that $T$. gondii IgG serointensity was positively associated with cardinal symptoms of depression in the Old Order Amish [24]. Links have also been identified between $T$. gondii IgG seropositivity or serointensity and suicidal behavior [25-29] and with an increased risk of traffic accidents [30-32].

Disturbances in sleep are more common and severe in those suffering from psychiatric conditions [33, 34], including schizophrenia [35-39], depression [40], bipolar disorder [41] and suicidal behavior [42-44], as well as car accidents [45], all of which, as stated above, have also been previously associated with $T$. gondii infection. We thus reasoned that sleep impairment might mediate, at least in part, the link between $T$. gondii infection and psychiatric disorders and behavioral dysregulation. Recently, we reported in a sample of Old Order Amish, no associations of $T$. gondii IgG seropositivity and serointensity with bedtime difficulties or daytime sleepiness [46].

Delayed sleep phase is associated with mood disturbances, such as unipolar depression and bipolar disorder [47]. Delayed timing and duration of sleep have been reported in bipolar depression [48, 49]. Moreover, severity of depressive symptoms is increased in those with delayed timing of sleep [50]. Sleep midpoint has also been reported to be delayed in those suffering from depression [51]. Given these data, we expected that later bedtime, mid-sleep time and wake-up time would be positively associated with $T$. gondii IgG serointensity or seropositivity.

Given that low-grade immune activation is known to alter sleep [52], together with the production by $T$. gondii of dopamine [53,54], a wakefulness-promoting neurotransmitter, we hypothesized that $T$. gondii would be associated with changes in the duration of sleep, delayed timing of sleep, problems maintaining sleep and increased daytime problems due to poor sleep.

\section{Methods}

\section{Study population}

Data were drawn from the Amish Wellness Study, which began in 2010 as part of a cardio-metabolic screening program for the Amish community in Lancaster County, 
PA, USA. Amish Research Clinic of the University of Maryland, Baltimore is located in Lancaster, PA, USA, and nurses from this clinic recruited our study subjects. The inclusion criteria included: belonging to the Old Order Amish Community, being over 18 years old, and for our sub-project - having responded to a variant of a sleep questionnaire containing the variables of interest, as well as having $T$. gondii IgG titers and seropositivity results from a sub-study nested in the Amish Wellness Study that focused on the environmental and genetic risk factors for T. gondii infection. Informed consent for the parent Amish Wellness study was obtained after a thorough explanation of the study by nursing staff and Amish liaisons (Old Order Amish women working to secure a culturally sensitive interface between the Amish community and the nursing and medical staff of the University of Maryland Baltimore Amish Research Clinic). The protocol for the parent Amish Wellness study was approved by the University of Maryland, Baltimore Institutional Review Board.

The study sample comprised 833 Old Order Amish adults [mean age (SD) $=44.28$ (16.99) years], which included 341 (40.94\%) men and 492 (59.06\%) women. They each responded to sleep questionnaires as part of a wellness screen in Lancaster, PA. The questionnaires were vetted by the Amish liaisons and by nursing personnel familiar with the Amish participants and culture.

Fasting blood samples were centrifuged for 25 minutes at $400 \mathrm{~g}$ and at $4^{\circ} \mathrm{C}$. Plasma was separated and stored at $-80^{\circ} \mathrm{C}$. To determine $T$. gondii serologic status, an enzyme-linked immunosorbent assay (ELISA) (IBL International, Männedorf, Switzerland) was used to test the plasma for IgG antibodies to T. gondii. The maximum dilution was 1:20. Status of each of the samples (i.e., seropositive or seronegative for infection with $T$. gondii) was defined by a predetermined cutoff value of $10 \mathrm{IU} / \mathrm{mL}$, with a mean coefficient of variation of $7 \%$, as reported by the ELISA manufacturer. When ELISA results indicated an equivocal concentration of $T$. gondii IgG (8 to $12 \mathrm{IU} /$ $\mathrm{mL}$ ), the test was repeated. If the level was under $8 \mathrm{IU} /$ $\mathrm{mL}$ or over $12 \mathrm{IU} / \mathrm{mL}$, it was considered seronegative or seropositive for infection with $T$. gondii, respectively. The individuals with persistent equivocal results were excluded from analysis.

Informed consent: Informed consent was obtained from all individuals included in this study.

Ethical approval: The research related to human use was in compliance with all the relevant national regulations, institutional policies and in accordance with the tenets of the Helsinki Declaration, and was approved by the authors' institutional review board or equivalent committee.

\section{Sleep questionnaire}

Selected questions were administered to measure our variables of interest, which were all analyzed in binary form. Sample sizes per variable differed due to the availability of $T$. gondii serology results in those subjects that had answered the sleep questionnaire. The questions "trouble staying asleep" (sleep maintenance), "problems during the day due to poor sleep" and "daytime sleepiness" were answered by 833 individuals, 455 (54.62\%) of which were T. gondii seropositive. Other questions regarding sleep that did not include the ones mentioned above (because of a change in questionnaire length due to logistical issues), were previously analyzed in relationship to markers of chronic T. gondii infection [46]. Bedtime and wake-up time were self-reported and the mid-sleep time was calculated. All sleep timings were converted to decimal hours for analysis.

\section{Statistical methods}

The relationship between $T$. gondii IgG seropositivity and transformed ranked answers from sleep questionnaires were analyzed using binary logistic regression, adjusted for age and sex (SAS version 9.3 SAS Institute Inc., Cary, NC, USA). The associations of T. gondii IgG titers with sleep duration and timing were analyzed using Linear Mixed Models adjusted for age, sex and family aggregation, using the Mixed Model Analysis for Pedigrees and Populations (MMAP) [55].

\section{Results}

Digitally transformed timing of average bedtime (SD) was 21.38 (0.54), and average wake-up time (SD) was 05.14 (0.72). Average mid-sleep time (SD) was 01.26 (0.49). In analog clock-time our participants' average bedtime was 9:22 PM and wake-up time was 5:08 AM.

\section{T. gondii seropositivity and sleep parameters}

There were 455 (54.61\%) T. gondii seropositive and 378 (45.39\%) T. gondii seronegative participants. Sleep variables in the overall sample and stratified by $T$. gondii seropositivity status are presented in Table 1.

After adjustment for age and sex, T. gondii seropositivity had a significant negative association with difficulty staying asleep [OR 0.79, 95\% CI: 0.68-0.92] and 
Table 1: Sleep parameters and T. gondii seropositive/seronegative status.

\begin{tabular}{|c|c|c|c|}
\hline Sleep parameters & All & T. gondii (+) & T. gondii (-) \\
\hline \multicolumn{4}{|c|}{ Trouble falling asleep $(n=829)$} \\
\hline No & $593(71.53 \%)$ & $330(39.81 \%)$ & $263(31.72)$ \\
\hline Yes & $236(28.47 \%)$ & $122(14.72 \%)$ & $114(13.75 \%)$ \\
\hline \multicolumn{4}{|c|}{ Trouble staying asleep $(n=833)$} \\
\hline No & $415(49.82 \%)$ & $239(28.69 \%)$ & $176(21.13 \%)$ \\
\hline Yes & $418(50.18 \%)$ & $216(25.93 \%)$ & $202(24.25 \%)$ \\
\hline \multicolumn{4}{|c|}{ Sleep quality $(n=828)$} \\
\hline Poor & $13(1.57 \%)$ & $5(0.60 \%)$ & $8(0.97 \%)$ \\
\hline Good & $815(98.43 \%)$ & $450(54.35 \%)$ & $365(44.08 \%)$ \\
\hline \multicolumn{4}{|c|}{ Problems during the day due to poor sleep $(n=833)$} \\
\hline No & $668(80.19 \%)$ & $381(45.74 \%)$ & $287(34.45 \%)$ \\
\hline Yes & $165(19.81 \%)$ & $74(8.88 \%)$ & $91(10.92 \%)$ \\
\hline \multicolumn{4}{|c|}{ Daytime sleepiness $(n=833)$} \\
\hline No & $337(40.46 \%)$ & $199(23.89 \%)$ & $138(16.57 \%)$ \\
\hline Yes & $496(59.54 \%)$ & $256(30.73 \%)$ & $240(28.81 \%)$ \\
\hline
\end{tabular}

problems during the day due to poor sleep [OR 0.77, 95\% CI: 0.64-0.92].

\section{T. gondii serointensity and sleep parameters}

T. gondii serointensity was significantly negatively associated with bedtime and mid-sleep time, and positively associated with sleep duration. Specifically, higher T. gondii serointensity was associated with earlier bedtime $(\beta=-0.04, p=0.0002)$, earlier mid-sleep time $(\beta$ $=-0.002, p=0.02)$ and longer sleep duration $(\beta=0.04$, $p=0.02)$. Wake-up time $(\beta=-0.0009, p=0.07)$ was not significantly changed with $T$. gondii serointensity (trend towards statistical significance).

\section{Discussion}

To our knowledge, the current study is the first to find a significant association between markers of $T$. gondii infection and sleep duration, timing and maintenance, as well as problems during the day due to poor sleep. The direction of these significant associations was, in part, surprising. Earlier, rather than later, bedtime and midsleep time, and longer rather than shorter sleep duration, were associated with higher $T$. gondii IgG titers. Moreover,
T. gondii IgG seropositive individuals reported less, rather than more, difficulties in maintaining sleep and had fewer problems during the day due to poor sleep.

One avenue by which $T$. gondii may influence sleep is directly through its endogenous production of dopamine $[53,54,56]$. Evidence indicates that there is a relationship between dopamine and sleep-wake cycles. Patients with Parkinson's disease, which reduces dopamine-producing neurons [57], often have excessive daytime sleepiness [58]. Nishino et al. (1998) demonstrated that dopaminespecific reuptake blockers increase wakefulness in normal and narcoleptic animals [59]. Wisor et al. (2001) reported increased wakefulness and reduction in NREM sleep in mice with the absence of the dopamine transporter gene [60]. A study done on dopamine $\left(D_{2}\right)$ receptor knockout mice showed decreased wakefulness, shorter wake periods, increased NREM sleep and increased stage transitions between being awake and NREM sleep [61]. Additionally in this study, sleep quality was also affected, as indicated by the lower delta activity, a component of deep sleep [61]. These data indicate the possibility that dopamine promotes wakefulness.

Chronic infection with $T$. gondii may also promote daytime wakefulness, as suggested by better self-reported tolerance of daytime consequences of poor sleep in our study sample. One way to explain the wakefulness effect is by dopamine's implication in homeostatic and 
circadian components of sleep/wake regulation, which in turn are involved in numerous interactions with the neural, endocrine and immunological systems. Dopamine projects from the ventral tegmental area and substantia nigra, both of which contain functional clocks that schedule activities in a circadian manner [62-64], and leads to the promotion of wakefulness $[58,59,61] . T$. gondii can alter dopamine production in several ways. Firstly, it possesses tyrosine hydroxylase [54], the ratelimiting enzyme in dopamine production [65]. Secondly, inflammation secondary to $T$. gondii infection may activate the kynurenine pathway, leading to decreased brain levels of kynurenic acid, higher levels of which have been reported to have an inverse relationship with dopamine levels [66]. Thirdly, immune activation (necessary to contain $T$. gondii in immunocompetent hosts) may also interact with dopaminergic processes by inducing alterations in the level of tyrosine, the precursor of dopamine [65]. A high phenylalanine:tyrosine ratio, resulting from inhibition of phenylalanine hydroxylase $[67,68]$, can be the consequence of Th1 activation, one of the central immune mechanisms responsible for containing $T$. gondii infection in immunocompetent hosts [69]. We recently reported how associations between $T$. gondii and aggression [70] or impulsivity [71], known to be in part modulated by dopaminergic pathways, interact with plasma peripheral levels of phenylalanine:tyrosine ratios.

Independent of dopaminergic mechanisms, as any microbial organism, $T$. gondii can promote sleep through the induction of the immune system. Immune pathways that aid in the containment of $T$. gondii, as well as other infections in the immunocompetent host, have also been implicated in sleep-wake regulation. For instance, levels of IL-12 and TNF, both proinflammatory cytokines that have been linked to the acute control of $T$. gondii infection [72-75], peak during sleep regardless of circadian factors [1]. The activation of TNF initiates sleep [76]. IL-10, an anti-inflammatory cytokine, prevents overly active immune response during infection [77] and also peaks during sleep [1]. It is possible that low-grade immune activation, potentially as a direct consequence of $T$. gondii infection, is involved in mediating the relationship of $T$. gondii IgG seropositivity and IgG titers with sleep duration and reduced problems in maintaining sleep. This could be evaluated in future longitudinal studies with multiple immune markers.

The evolutionary pull of survival may, in part, be driving a parasite's ability to protect sleep in its host thereby benefitting both host and pathogen. The host who sleeps longer with minimal nightly interruptions can have better health and may be more equipped to contain a pathogen through energy conservation $[1,3]$, ultimately reducing the chance of death, other than via predation by a representative of the cat family. Moreover, specifically for T. gondii, increased activity and reduced neophobia during wakefulness (as described in infected rodents) [78], may increase chances of rodent predation by a feline, thus completing the parasite's life-cycle.

An alternative possibility is that some strains of $T$. gondii act as microbial "Old Friends" with immunoregulatory capabilities [79] and subsequently, provide their intermediate host with a mutually beneficial relationship, such as less problems related to sleep, healthier timing and duration of sleep, with a greater longevity of the host, as well as a longer exposure to predation by the members of the cat family, eventually, resulting in a greater chance for $T$. gondii to reproduce. This would not be an isolated clinical link as it is known, for example, that $T$. gondii infection is associated with lower incidence of allergy and asthma [80, 81], conditions that have also been linked to poor sleep [82, 83]. Although T. gondii's common route of infection in humans is through the intestinal wall, there are relatively few studies focused on chronic toxoplasmosis and long term consequences on the gut microbiome, and further on immune function studies in human or nonhuman animals. In one small study, chronic $T$. gondii-infected mice did have an increase in gut immunomodulatory bacteria compared to uninfected mice [84].

\section{Strengths of the study}

The Amish have a relatively homogeneous lifestyle [85] and have high rates of T. gondii seropositivity [86]. Limited alcohol and substance use consumption, as well as the absence of exposure to bright or blue artificial lighting from television sets, cell phones and computers [85] that alter the circadian rhythm and perturb sleep [87], are additional strengths of our study.

\section{Limitations}

We had a cross-sectional design, did not adjust for multiple comparisons (with only the negative association between bedtime and $T$. gondii IgG serointensity being able to resist a full adjustment for multiple comparisons, post hoc), and used self-report sleep measures rather than objective measures. Since, the study was done in the Old Order Amish, the generalizability of the results is limited. 
We have not accounted for markers of inflammation and have not analyzed IgM and other markers to differentiate acute from chronic $T$. gondii infection. We cannot be sure if the lack of a significant association of titers with wake-up time is a result of more dominant environmental (likely occupational demands) factors, in comparison to midsleep and bedtime, or it being a consequence of a type II error due to a limited sample size, resulting in a statistical trend rather than achieving statistical significance.

In sum, T. gondii IgG seropositivity is associated with healthier sleep profiles, specifically having fewer problems maintaining sleep and fewer daytime problems due to poor sleep, and higher T. gondii IgG titers are associated with earlier bedtime and mid-sleep time, and longer sleep duration. Thus, there is no current evidence to support deleterious associations between $T$. gondii infection and sleep; hence, there are no grounds to continue to expect that sleep impairment could mediate the predictive associations between $T$. gondii infection and mental illness, suicidal behavior, or traffic accidents. Our results need to be replicated with longitudinal designs using objective measures of sleep. Future studies should also investigate potential immune mechanisms mediating these associations by measuring a comprehensive panel of inflammatory markers. For instance, given the high seroprevalence of $T$. gondii and considerable rate of seroconversion in the Amish, we can focus on measures of sleep continuity and timing on actigraphic tracing, or even polysomnography at baseline in T. gondii seronegative individuals, and then compare sleep patterns and differences from baseline in individuals who seroconverted versus those who remained seronegative. At several (at least two) time points, sleep questionnaires, sleep latency, wake-maintenance tests, sleep onset and offset and tests of alertness, such as the psychomotor vigilance tests [88], could be related to T. gondii seropositive status, serointensity, cytokine profiles and potential structural and functional neuroimaging. Additionally, circadian markers, such as dim light saliva melatonin onset [89, 90], could lead us closer to understanding the direction of causality and mechanisms of the unexpected associations revealed by our cross-sectional study.

Acknowledgments: We would like to thank the University of Maryland, Joint Institute for Food Safety and Applied Nutrition and the U.S. Food and Drug Administration (U.S. FDA) for their support through their cooperative agreement FDU.001418 (PI, Postolache). We also acknowledge our gratitude to the participants for their time and willingness to participate in the study. We thank the entire staff of the University of Maryland
School of Medicine, Amish Research Clinic, Lancaster, PA, USA. Additional support was received from the MidAtlantic Nutrition Obesity Research Center (NORC) Pilot \& Feasibility Project (Postolache, PI), a sub-award of the parent grant P30DK072488 (Simeon I. Taylor, Program Director) and the Merit Award 1 I01 CX001310-01 from CSR\&D/Veterans Affairs Administration (PI, Postolache). The results and interpretations provided represent opinions of the authors and not necessarily the official positions of the Department of Defense, United States Air Force, VA, NIH or US-FDA.

Conflict of interest: Authors state no conflict of interest.

\section{References}

1. Ibarra-Coronado EG, Pantaleón-Martínez AM, VelazquézMoctezuma J, Prospéro-García O, Méndez-Díaz M, Pérez-Tapia $M$, et al. The bidirectional relationship between sleep and immunity against infections. J Immunol Res. 2015;2015:678164.

2. Buguet A, Bisser S, Josenando T, Chapotot F, Cespuglio R. Sleep structure: a new diagnostic tool for stage determination in sleeping sickness. Acta Trop. 2005;93(1):107-17.

3. Preston BT, Capellini I, McNamara P, Barton RA, Nunn CL. Parasite resistance and the adaptive significance of sleep. BMC Evol Biol. 2009;9:7.

4. Opp MR. Sleeping to fuel the immune system: mammalian sleep and resistance to parasites. BMC Evol Biol. 2009;9:8.

5. Jackson $\mathrm{MH}$, Hutchison WM. The prevalence and source of Toxoplasma infection in the environment. Adv Parasitol. 1989;28:55-105.

6. Jones J, Kruszon-Moran D, Rivera H, Price C, Wilkins P. Toxoplasma gondii seroprevalence in the United States 20092010 and comparison with the past two decades. Am J Trop Med Hyg. 2014;90(6):1135-9.

7. Frenkel J, Dubey J, Miller N. Toxoplasma gondii in cats: fecal stages identified as coccidian oocysts. Science. 1970;167(3919):893-6.

8. Elmore S, Jones J, Conrad P, Patton S, DS L, Dubey J. Toxoplasma gondii: Epidemiology, feline clinical aspects, and prevention. Trends Parasitol. 2010;4(26):190-6.

9. Jones J, Kruszon-Mora D, Wilson M, McQuillan G, Navin T, McAuley J. Toxoplasma gondii infection in the United States: Seroprevalence and risk factors. Am J Epidemiol. 2001;154(4):357-65.

10. Baril L, Ancelle T, Goulet V, Thulliez P, Tirad-Fleury V, Carme B. Risk factors for Toxoplasma infection in pregnancy: a casecontrol study in France. Scand J Infect Dis. 1999;31(3):305-9.

11. Weigel R, Dubey J, Siegel A. Risk factors for infection with Toxoplasma gondii for residents and workers on swine farms in Illinois. Am J Trop Med Hyg. 1999;60(5):793-8.

12. Kapperud G, Jenum P, Stray-Pederson B, Melby K, Eskild A, Eng J. Risk factors for Toxoplasma gondii infection in pregnancy. Results of a prospective case-control study in Norway. Am J Epidemiol. 1996;144(4):405-12. 
13. Cook A, Gilbert R, Buffolano W, Zufferey J, Petersen E, Jenum $P$, et al. Sources of toxoplasma infection in pregnant women: European multicentre case-control study. European Research Network on Congenital Toxoplasmosis. BMJ. 2000;321(7254):142-7.

14. Pittman KJ, Knoll LJ. Long-Term Relationships: The complicated interplay between the host and the developmental stages of toxoplasma gondii during acute and chronic infections. Microbiol Mol Biol Rev. 2015;79(4):387-401.

15. Donovick PJ, Burright RG. The consequences of parasitic infection for the behavior of the mammalian host. Environ Health Perspect. 1987;73:247-50.

16. Berdoy M, Webster JP, Macdonald DW. Fatal attraction in rats infected with Toxoplasma gondii. Proc Biol Sci. 2000;267(1452):1591-4.

17. Kannan G, Moldovan K, Xiao JC, Yolken RH, Jones-Brando L, Pletnikov MV. Toxoplasma gondii strain-dependent effects on mouse behaviour. Folia Parasitol (Praha). 2010;57(2):151-5.

18. Bohne W, Heesemann J, Gross U. Reduced replication of Toxoplasma gondii is necessary for induction of bradyzoitespecific antigens: a possible role for nitric oxide in triggering stage conversion. Infect Immun. 1994;62(5):1761-7.

19. Soete M, Dubremetz J. Toxoplasma gondii: kinetics of stagespecific protein expression during tachyzoite-bradyzoite conversion in vitro. Curr Top Microbiol Immunol. 1996;219:7680.

20. Denkers E, Gazzinelli R. Regulation and function of T-cellmediated immunity during Toxoplasma gondii infection. Clin Microbiol Rev. 1998;11(4):569-88.

21. Torrey EF, Bartko JJ, Lun ZR, Yolken RH. Antibodies to Toxoplasma gondii in patients with schizophrenia: a metaanalysis. Schizophr Bull. 2007;33(3):729-36.

22. Yolken RH, Bachmann S, Ruslanova I, Lillehoj E, Ford G, Torrey EF, et al. Antibodies to Toxoplasma gondii in individuals with first-episode schizophrenia. Clin Infect Dis. 2001;32(5):842-4.

23. Sutterland A, Fond G, Kuin A, Koeter M, Lutter R, Gool T, et al. Beyond the association. Toxoplasma gondii in schizophrenia, bipolar disorder, and addiction: systematic review and metaanalysis. Acta Psychiatr Scand. 2015;132(3):161-79.

24. Wadhawan A, Dagdag A, Duffy A, Daue ML, Ryan KA, Brenner LA, et al. Positive association between Toxoplasma gondii IgG serointensity and current dysphoria/hopelessness scores in the Old Order Amish: a preliminary study. Pteridines. 2017;28(3-4):185-94.

25. Arling TA, Yolken RH, Lapidus M, Langenberg P, Dickerson FB, Zimmerman SA, et al. Toxoplasma gondii antibody titers and history of suicide attempts in patients with recurrent mood disorders. J Nerv Ment Dis. 2009;197(12):905-8.

26. Ling VJ, Lester D, Mortensen PB, Langenberg PW, Postolache TT. Toxoplasma gondii seropositivity and suicide rates in women. J Nerv Ment Dis. 2011;199(7):440-4.

27. Okusaga O, Postolache TT. Frontiers in neuroscience. Toxoplasma gondii, the immune system, and suicidal behavior. In: Dwivedi Y, editor. The Neurobiological Basis of Suicide. Boca Raton (FL): CRC Press/Taylor \& Francis Llc.; 2012.

28. Pedersen MG, Mortensen PB, Norgaard-Pedersen B, Postolache TT. Toxoplasma gondii infection and self-directed violence in mothers. Arch Gen Psychiatry. 2012;69(11):1123-30.

29. Zhang Y, Traskman-Bendz L, Janelidze S, Langenberg P, Saleh A, Constantine N, et al. Toxoplasma gondii immunoglobulin G antibodies and nonfatal suicidal self-directed violence. J Clin Psychiatry. 2012;73(8):1069-76.

30. Flegr J, Havlícek J, Kodym P, Malý M, Smahel Z. Increased risk of traffic accidents in subjects with latent toxoplasmosis: a retrospective case-control study. BMC Infect Dis. 2002;2(1):11.

31. Kocazeybek B, Oner YA, Turksoy R, Babur C, Cakan H, Sahip $\mathrm{N}$, et al. Higher prevalence of toxoplasmosis in victims of traffic accidents suggest increased risk of traffic accident in Toxoplasma-infected inhabitants of Istanbul and its suburbs. Forensic Sci Int. 2009;187(1):103-8.

32. Yereli K, Balcioğlu IC, Özbilgin A. Is Toxoplasma gondii a potential risk for traffic accidents in Turkey? Forensic Sci Int. 2006;163(1):34-7.

33. Kamphuis J, Karsten J, de Weerd A, Lancel M. Sleep disturbances in a clinical forensic psychiatric population. Sleep Med. 2013;14(11):1164-9.

34. Spiegelhalder K, Regen W, Nanovska S, Baglioni C, Riemann D. Comorbid sleep disorders in neuropsychiatric disorders across the life cycle. Curr Psychiatry Rep. 2013;15(6):364.

35. Anderson KN, Bradley AJ. Sleep disturbance in mental health problems and neurodegenerative disease. Nat Sci Sleep. 2013;5:61-75.

36. Benson KL. Sleep in schizophrenia: Pathology and treatment. Sleep Med Clin. 2015;10(1):49-55.

37. Cohrs S. Sleep disturbances in patients with schizophrenia: impact and effect of antipsychotics. CNS Drugs. 2008;22(11):939-62.

38. Klingaman EA, Palmer-Bacon J, Bennett ME, Rowland LM. Sleep disorders among people with schizophrenia: Emerging research. Curr Psychiatry Rep. 2015;17(10):79.

39. Palmese LB, DeGeorge PC, Ratliff JC, Srihari VH, Wexler BE, Krystal AD, et al. Insomnia is frequent in schizophrenia and associated with night eating and obesity. Schizophr Res. 2011;133(1-3):238-43.

40. Nutt D, Wilson S, Paterson L. Sleep disorders as core symptoms of depression. Dialogues Clin Neurosci. 2008;10(3):329-36.

41. Gold AK, Sylvia LG. The role of sleep in bipolar disorder. Nat Sci Sleep. 2016;8:207-14.

42. Benard V, Etain B, Vaiva G, Boudebesse C, Yeim S, Benizri C, et al. Sleep and circadian rhythms as possible trait markers of suicide attempt in bipolar disorders: An actigraphy study. J Affect Disord. 2019;244:1-8.

43. Lin HT, Lai CH, Perng HJ, Chung CH, Wang CC, Chen WL, et al. Insomnia as an independent predictor of suicide attempts: $\mathrm{a}$ nationwide population-based retrospective cohort study. BMC Psychiatry. 2018;18(1):117.

44. Pigeon WR, Britton PC, Ilgen MA, Chapman B, Conner KR. Sleep disturbance preceding suicide among veterans. Am J Public Health. 2012;102 Suppl 1(Suppl 1):S93-7.

45. Barger LK, Rajaratnam SM, Wang W, O’Brien CS, Sullivan JP, Qadri S, et al. Common sleep disorders increase risk of motor vehicle crashes and adverse health outcomes in firefighters. J Clin Sleep Med. 2015;11(3):233-40.

46. Ahmad Z, Moustafa YW, Stiller JW, Pavlovich MA, Raheja UK, Gragnoli C, et al. Sleep onset insomnia, daytime sleepiness and sleep duration in relationship to Toxoplasma gondii IgG seropositivity and serointensity. Pteridines. 2017;28(3-4):195204.

47. Steinan MK, Morken G, Lagerberg TV, Melle I, Andreassen OA, Vaaler AE, et al. Delayed sleep phase: An important circadian 
subtype of sleep disturbance in bipolar disorders. J Affect Disord. 2016;191:156-63.

48. Geoffroy PA, Scott J, Boudebesse C, Lajnef M, Henry C, Leboyer $M$, et al. Sleep in patients with remitted bipolar disorders: A meta-analysis of actigraphy studies. Acta Psychiatr Scand. 2015;131(2):89-99.

49. Ritter PS, Marx C, Lewtschenko N, Pfeiffer S, Leopold K, Bauer $\mathrm{M}$, et al. The characteristics of sleep in patients with manifest bipolar disorder, subjects at high risk of developing the disease and healthy controls. J Neural Transm. 2012;119(10):1173-84.

50. Murray JM, Sletten TL, Magee M, Gordon C, Lovato N, Bartlett DJ, et al. Prevalence of circadian misalignment and its association with depressive symptoms in delayed sleep phase disorder. Sleep. 2017;40(1).

51. Hasler BP, Buysse DJ, Kupfer DJ, Germain A. Phase relationships between core body temperature, melatonin, and sleep are associated with depression severity: Further evidence for circadian misalignment in non-seasonal depression. Psychiatry Res. 2010;178(1):205-7.

52. Krueger JM. The role of cytokines in sleep regulation. Curr Pharm Des. 2008;14(32):3408-16.

53. Gaskell EA, Smith JE, Pinney JW, Westhead DR, McConkey GA. A unique dual activity amino acid hydroxylase in Toxoplasma gondii. PLoS One. 2009;4(3).

54. Prandovszky E, Gaskell E, Martin H, Dubey J, Webster JP, McConkey GA. The neurotropic parasite Toxoplasma gondii increases dopamine metabolism. PloS One. 2011;6(9).

55. O’Connell J. MMAP User Guide 2014 [Available from: http://edn. som.umaryland.edu/mmap/index.php.

56. Webster JP. Rats, cats, people and parasites: The impact of latent toxoplasmosis on behaviour. Microbes Infect. 2001;3(12):1037-45.

57. Lotharius J, Brundin P. Pathogenesis of Parkinson's disease: dopamine, vesicles and $\alpha$-synuclein. Nat Rev Neurosci 2002;3:932-42.

58. Arnulf I, Konofal E, Merino-Andreu M, Houeto J, Mesnage V, Welter M, et al. Parkinson's disease and sleepiness an integral part of PD. Neurology. 2002;58(7):1019-24.

59. Nishino S, Mao J, Sampathkumaran R, Shelton J. Increased dopaminergic transmission mediates the wake-promoting effects of CNS stimulants. Sleep Res Online. 1998;1(1):49-61.

60. Wisor J, Nishino S, Sora I, Uhi G, Mignot E, Edgar D. Dopaminergic role in stimulant-induced wakefulness. J Neurosci. 2001;5:1787-94.

61. Qu WM, Xu XH, Yan MM, Wang YQ, Urade Y, Huang ZL. Essential role of dopamine D2 receptor in the maintenance of wakefulness, but not in homeostatic regulation of sleep, in mice. J Neurosci. 2010;30(12):4382-9.

62. Chung S, Lee EJ, Yun S, Choe HK, Park S-B, Son HJ, et al. Impact of circadian nuclear receptor REV-ERB $\alpha$ on midbrain dopamine production and mood regulation. Cell. 2014;157(4):858-68.

63. Webb IC, Baltazar RM, Wang X, Pitchers KK, Coolen LM, Lehman $M N$. Diurnal variations in natural and drug reward, mesolimbic tyrosine hydroxylase, and clock gene expression in the male rat. J Biol Rhythms. 2009;24(6):465-76.

64. Weber M, Lauterburg T, Tobler I, Burgunder J-M. Circadian patterns of neurotransmitter related gene expression in motor regions of the rat brain. Neurosci Lett. 2004;358(1):17-20.
65. Daubner SC, Le T, Wang S. Tyrosine hydroxylase and regulation of dopamine synthesis. Arch Biochem Biophys. 2011;508(1):112.

66. Wu HQ, Rassoulpour A, Schwarcz R. Kynurenic acid leads, dopamine follows: A new case of volume transmission in the brain? J Neural Transm (Vienna). 2007;114(1):33-41.

67. Neurauter G, Grahmann A, Kliber M, Zeimet A, Ledochowski $M$, Sperner-Unterweger $B$, et al. Serum phenylalanine concentrations in patients with ovarian carcinoma correlate with concentrations of immune activation markers and of isoprostane-8. Cancer Lett. 2008;272(1):141-7.

68. Capuron L, Schroecksnadel S, Feart C, Aubert A, Higueret D, Barberger-Gateau P, et al. Chronic low-grade inflammation in elderly persons is associated with altered tryptophan and tyrosine metabolism: role in neuropsychiatric symptoms. Biol Psychiatry. 2011;70(2):175-82.

69. Spellberg B, Edwards JE, Jr. Type 1/Type 2 immunity in infectious diseases. Clin Infect Dis. 2001;32(1):76-102.

70. Mathai AJ, Lowry CA, Cook TB, Brenner LA, Brundin L, Groer MW, et al. Reciprocal moderation by Toxoplasma gondii seropositivity and blood phenylalanine - tyrosine ratio of their associations with trait aggression. Pteridines. 2016;27(3-4):7785.

71. Peng X, Brenner LA, Mathai AJ, Cook TB, Fuchs D, Postolache $\mathrm{N}$, et al. Moderation of the relationship between Toxoplasma gondii seropositivity and trait impulsivity in younger men by the phenylalanine-tyrosine ratio. Psychiatry Res. 2018;270:992-1000. doi:10.1016/j.psychres.2018.03.045.

72. Gazzinelli RT, Hayashi S, Wysocka M, Carrera L, Kuhn R, Muller W, et al. Role of IL-12 in the initiation of cell mediated immunity by Toxoplasma gondii and its regulation by IL-10 and nitric oxide. J Eukaryot Microbiol. 1994;41(5):9s.

73. Sher A, Oswald IP, Hieny S, Gazzinelli RT. Toxoplasma gondii induces a T-independent IFN-gamma response in natural killer cells that requires both adherent accessory cells and tumor necrosis factor-alpha. J Immunol. 1993;150(9):3982-9.

74. Yap GS, Scharton-Kersten T, Charest H, Sher A. Decreased resistance of TNF receptor $\mathrm{p} 55$ - and $\mathrm{p} 75$-deficient mice to chronic toxoplasmosis despite normal activation of inducible nitric oxide synthase in vivo. J Immunol. 1998;160(3):1340-5.

75. Khan IA, Matsuura T, Kasper LH. Interleukin-12 enhances murine survival against acute toxoplasmosis. Infect Immun. 1994;62(5):1639-42.

76. Lorton D, Lubahn CL, Estus C, Millar BA, Carter JL, Wood CA, et al. Bidirectional communication between the brain and the immune system: Implications for physiological sleep and disorders with disrupted sleep. Neuroimmunomodulation. 2006;13(5-6):357-74.

77. Gazzinelli RT, Wysocka M, Hieny S, Scharton-Kersten T, Cheever A, Kuhn R, et al. In the absence of endogenous IL-10, mice acutely infected with Toxoplasma gondii succumb to a lethal immune response dependent on CD4+ T cells and accompanied by overproduction of IL-12, IFN-gamma and TNF-alpha. J Immunol. 1996;157(2):798-805.

78. Webster J. The effect of toxoplasma gondii on animal behavior: Playing cat and mouse. Schizophr Bull. 2007;33:752-6.

79. Rook GA, Lowry CA, Raison CL. Microbial 'Old Friends', immunoregulation and stress resilience. Evol Med Public Health. 2013;2013(1):46-64. 
80. Fenoy I, Giovannoni M, Batalla E, Martin V, Frank FM, Piazzon I, et al. Toxoplasma gondii infection blocks the development of allergic airway inflammation in BALB/c mice. Clin Exp Immunol. 2009;155(2):275-84.

81. Fenoy IM, Sanchez VR, Soto AS, Picchio MS, Martin V, Goldman A. Toxoplasma gondii infection modulate systemic allergic immune response in BALB/c mice. Exp Parasitol. 2015;154:47 50.

82. Fagnano M, Bayer A, Isensee C, Hernandez T, Halterman J. Nocturnal asthma symptoms and poor sleep quality among urban school children with asthma. Acad Pediatr 2011;11:4939.

83. Kakumanu S, Glass C, Craig T. Poor sleep and daytime somnolence in allergic rhinitis: significance of nasal congestion. Am J Respir Med. 2002;1(3):195-200.

84. Prandovszky E, Li Y, Sabunciyan S, Steinfeldt C, Avalos L, Gressitt K, et al. Toxoplasma gondii-induced long-term changes in the upper intestinal microflora during the chronic stage of infection. Scientifica (Cairo). 2018. Article ID 2308619:1-11.

85. Kraybill D, Johnson-Weiner K, Nolt S. The Amish. Baltimore, MD: Johns Hopkins University Press; 2013.

86. Duffy A, Pavlovich M, Groer M, Peng X, Ryan K, Nijjar G, et al. 914. Toxoplasma gondii serointensity and seropositivity and their heritability and household-related associations in the old order Amish. Biol Psychiatry. 2017;81(10):S369-S70.

87. Cajochen C, Frey S, Anders D, Spati J, Bues M, Pross A, et al. Evening exposure to a light-emitting diodes (LED)-backlit computer screen affects circadian physiology and cognitive performance. J Appl Physiol. 2011;110(5):1432-8.

88. Dinges D, Powell J. Microcomputer analyses of performance on a portable, simple visual RT task during sustained operations. Behav Res Meth Instr Comp. 1985;17(6):652-5.

89. Lewy A, Sack R. The dim light melatonin onset as a marker for circadian phase position. Chronobiol Int. 1989;6(1):93-102.

90. Lewy A, Cutler N, Sack R. The endogenous melatonin profile as a marker for circadian phase position. J Biol Rhythms. 1999;14:227-36. 